\title{
Design and Analysis of an Ultrawide-Band Distributed CMOS Mixer
}

\author{
Amin Q. Safarian, Student Member, IEEE, Ahmad Yazdi, Student Member, IEEE, and Payam Heydari, Member, IEEE
}

\begin{abstract}
This paper presents the design and analysis of a novel distributed CMOS mixer for ultrawide-band (UWB) receivers. To achieve the UWB RF frequency range required for the UWB communications, the proposed mixer incorporates artificial inductance-capicitance $(L C)$ delay lines in radio frequency $(\mathrm{RF})$, local oscillator (LO), and intermediate frequency signal paths, and single-balanced mixer cells that are distributed along these $L C$ circuits. Closed-form analytical model for the conversion gain of the mixer is presented. Furthermore, a comprehensive noise analysis of the proposed distributed mixer is carried out, which includes calculation of the mixer noise figure (NF) and derivation of the optimum number of stages, $n$, minimizing the NF. The designed mixer is capable of covering the RF and LO frequencies over a wide range of frequencies from 3.1-8.72 GHz. A two-stage distributed mixer has been fabricated in a $0.18-\mu \mathrm{m}$ CMOS process. Experiments show a conversion gain of more than $2.5 \mathrm{~dB}$ for the entire range of the frequencies. The dc power consumption is $10.4 \mathrm{~mW}$.
\end{abstract}

Index Terms-CMOS radio frequency, distributed circuit, gain-bandwidth, impulse-radio, mixer, multiband, noise figure (NF), ultrawide-band (UWB), wireless communications.

\section{INTRODUCTION}

$\mathbf{U}$ LTRAWIDE-BAND (UWB) wireless broadcasts are capable of carrying huge amounts of data up to $250 \mathrm{ft}$ with extremely little transmit power and high immunity to interference and noise. Furthermore, the spread spectrum characteristics of UWB wireless systems, and the ability of the UWB wireless receivers to highly resolve the signal in multi-path fading channels due to the nature of the short duration transmitting impulse signals make the UWB systems a desirable wireless system of choice in a wide variety of high-rate, short- to medium-range communications. The ability to also locate objects to within one inch attracts the military, law-enforcement, and rescue agencies. Other applications include the broadband sensing using active sensor networks and collision avoidance. The circuit techniques that are used to realize different circuit components in a UWB transceiver are quite different from those proposed in current narrow bandwidth radio frequency (RF) technology. This notion provides an incentive to investigate the design of novel circuit topologies that achieve a gain-for-delay-tradeoff without affecting bandwidth, and thus operate at substantially higher frequencies than conventional circuits.

Manuscript received September 4, 2003; revised May 11, 2004 and September 28, 2004.

The authors are with the University of California, Irvine, CA 92697-2625

USA (e-mail: safariaa@uci.edu; payam@ece.uci.edu).

Digital Object Identifier 10.1109/TVLSI.2005.844288
One of the key circuit components in a UWB receiver is the front-end UWB mixer [17]. The introduction of traveling wave concept has provided very high frequency techniques to implement broadband integrated circuits. Distributed integrated circuits such as distributed amplifiers [8], [9], [6], [5], and distributed oscillators [8], [1] all employ actual or artificial transmission lines (T-lines) to achieve ultrawide bandwidth. As a fundamental property, integrated circuits incorporating on-chip transmission lines trade delay for bandwidth [10]. The idea of the distributed circuit topology can be utilized to design UWB mixers. A dual gate distributed mixer in HEMT technology was first presented in [4].

While using dual gate transistor to provide RF and local oscillator (LO) signal paths, this mixer exhibits a poor return loss. Recently, [3] has proposed a wideband GaAs PHEMT distributed mixer. The proposed mixer can operate over a wideband RF input of 3-33 GHz. It, however, suffers from a lower conversion gain, high susceptibility to the environmental noise such as inductive noise and power/ground bounce, and high power consumption. Most importantly, the mixer operation in [3] can only be achieved under a precisely equal LO and RF amplitude, which poses a severe limitation on the proposed mixer circuit.

In this paper, the systematic design and analysis of a novel distributed mixer in standard CMOS process is presented. The circuit utilizes a bank of identical active current commuting mixers, where the input and output terminals of each mixer is connected to the tap points of artificial transmission lines. In the current design, each cell constitutes a fully differential single-balanced circuit, thereby showing a robust performance in the presence of the common-mode noise. In each cell, the intermediate frequency (IF) component is formed by multiplying the input RF voltage by a periodic waveform running at the LO frequency. It will be shown that if the phase constant of the artificial T-lines are designed properly, the IF output of each cell will then be in-phase with other IF components coming from the other cells. Therefore, the IF output of each cell constructively adds up together at the output terminal of the T-line. As a consequence, this mixer achieves a better conversion gain over a wide range of frequencies compared to the conventional single-balanced mixer.

The paper is organized as follows. Section II discusses the operating principle of the proposed mixer. This section also encompasses a detailed analysis of the distributed mixer as well as the analytical derivation of a closed-form expression for the conversion gain. In Section III, the noise analysis of the mixer is presented while accounting for the noise contributions of all the intrinsic sources within each cell as well as the ones due to 


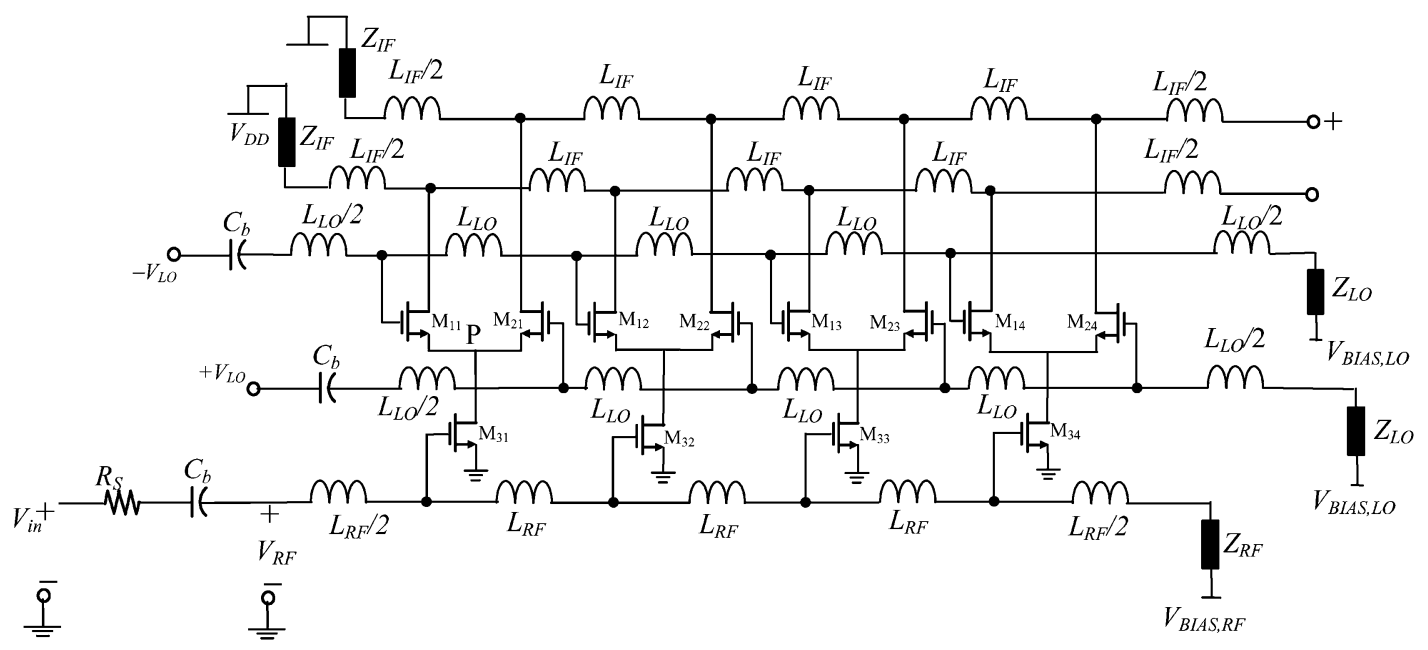

Fig. 1. Circuit schematic of the novel UWB mixer.

other cells propagating through the delay lines. Section IV includes simulation results of this mixer in the presence of noisy power supply and ground rails and a comparison with recently published results on distributed mixers. Finally, concluding remarks are provided in Section V.

\section{A Novel Distributed MiXer}

Recent advances in high-speed integrated circuits and continuous scaling of minimum feature sizes of devices have increased the interest in on-chip transmission lines (T-lines) as both parasitic components [7], and useful circuit components to achieve higher speeds of operation. Integrated circuits incorporating on-chip transmission lines trade propagation delay for the signal bandwidth. In the broadband systems the delay is more tolerable than limited bandwidth, because it can be calibrated using the delay prediction circuits, which justifies the use of distributed circuits in these applications. In the frequency domain, one source of bandwidth limitation in conventional analog circuits is the drop in input impedance of the circuits as frequency increases. In a distributed circuit, the transistor's input capacitance is absorbed into the transmission line [10]. Hence, until the cutoff frequency of the T-line itself is approached, the input impedance (and the bandwidth to a certain degree) remains constant.

The aforementioned features of the monolithic T-lines are utilized to design a novel distributed mixer whose circuit schematic is depicted in Fig. 1. The number of stages in the circuit schematic of Fig. 1 is arbitrarily chosen to be four. Each cell is a single-balanced mixer. However, other topologies of current commuting mixers can also be incorporated. The current tail transistors (M31, M32, M33, and M34) are identically matched. The switch pair transistors have the same geometry and biasing conditions. The output and input signals to each single-balanced cell are connected to the tap points of constituent artificial transmission lines.

\section{A. Operation Principle}

To include the most general scenario, consider a mixer consisting of $n$ single-balanced cells along the RF, LO, and IF Tlines (a 4-stage example is depicted in Fig. 1).

The artificial transmission lines are realized with inductance-capicitance $(L C)$ ladder networks where inductors are implemented using on-chip spirals, and capacitors are the parasitic capacitances of the MOS transistors that are connected to $L C$ delay lines. The load impedance is matched to the characteristic impedance of the $L C$ delay line. The RF voltage across the input terminal propagates through the RF T-line, and at tap-point along the RF T-line appears as the gate-source voltage to the current tail of each differential pair, producing a current proportional to the input RF signal. The differential-pair is driven by a large signal LO voltage, thereby acting as a differential voltage-controlled switch pair modulating the current tail. The output current of each mixing cell travels both in forward and reveres directions through each IF T-line. As discussed later in this Section, under a specific relationship among the phase velocities of RF, LO, and IF T-lines, the resultant IF signals traveling toward the output ports constructively add to the IF signals of subsequent mixer cells at corresponding tap points. On the other hand, the reverse traveling waves are out of phase, thereby canceling each other at each tap-point. The reverse traveling IF signals arriving at the IF T-lines are absorbed by the IF matched terminations [9], [11].

To derive closed-form analytical models for conversion gain and the noise figure (NF), a simplified unilateral model is used for the MOSFET device, as depicted in Fig. 2, where $r_{g}$ is the polysilicon gate resistance and $C_{\mathrm{gs}}$ is the gate-channel capacitance. $C_{d}$ and $r_{\mathrm{ds}}$ are the drain junction capacitance and on-resistance of the device, respectively. Additional coupling between T-lines due to the presence of drain-gate capacitance $\left(C_{\mathrm{gd}}\right)$ have been ignored, for the sake of simplification.

The voltage across the gate terminal of each current tail produces the small-signal RF current at the drain of the current tail. The RF current of the $k$ th single-balanced cell can be written as

$$
I_{\mathrm{RF}, k}=g_{m \mathrm{RF}} \cdot V_{i k} \quad k=1, \ldots, n
$$




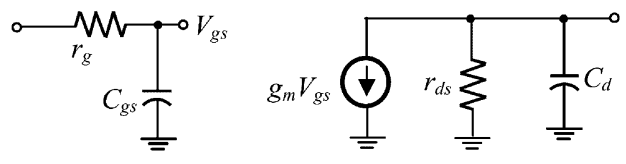

Fig. 2. Simplified unilateral small-signal model of the MOS device.

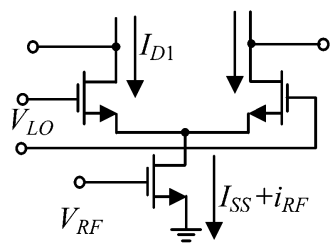

Fig. 3. Single-balanced mixer cell.

where $g_{m \mathrm{RF}}$ is the small-signal transconductance of the current tail transistors, and $V_{i k}$ is the gate-source voltage of the $k$ th section, which is obtained as follows [11]

$$
V_{i k}=\frac{V_{\mathrm{RF}} e^{-\left(k-\frac{1}{2}\right) \gamma_{\mathrm{RF}}-j \tan ^{-1}\left(\frac{\omega_{\mathrm{RF}}}{\omega_{g}}\right)}}{\left[1+\left(\frac{\omega_{\mathrm{RF}}}{\omega_{g} \mathrm{RF}}\right)^{2}\right]^{1 / 2}\left[1-\left(\frac{\omega_{\mathrm{RF}}}{\omega_{C}}\right)^{2}\right]}
$$

where $V_{\mathrm{RF}}$ is the input voltage, and $\gamma_{R F}=\alpha_{\mathrm{RF}}+j \beta_{\mathrm{RF}}$ is the propagation constant of the RF T-line. $\alpha_{\mathrm{RF}}$ and $\beta_{\mathrm{RF}}$ represent the attenuation and phase constants of the RF T-line, respectively. $\omega_{g \mathrm{RF}}=1 / r_{g \mathrm{RF}} C_{\mathrm{gs} \mathrm{RF}}$ is the input time constant of each transistor in the current tail of each mixer cell, $\omega_{C}$ is the cutoff frequency of the RF, LO, and IF T-lines (if the $L C$ delay lines are identical), and $\omega_{\mathrm{RF}}$ is the RF input frequency. Since a large amplitude differential LO signal is applied to switch-pair transistors, the bias point and hence the transconductance of those transistors is not fixed but varies periodically [2]. The output current of each single-balanced cell is a function of the instantaneous LO voltage $\left(V_{\mathrm{LO}}\right)$ at the gate terminals of switch-pair transistors and the RF tail current $i_{\mathrm{RF}}(t)$. The LO and RF input voltages to each mixer cell, which are the delayed replicas of the input LO and RF sources, are provided by LO and RF T-lines, respectively. Assuming the propagation phase constants of RF and LO line segments between any tap-points to be $\beta_{\mathrm{RF}}$ and $\beta_{\mathrm{LO}}$, respectively, the differential current at the output of the $k$ th single-balanced cell (cf. Fig. 3 ) is $(k=1, \ldots, n)$

$i_{o, k}(t)=i_{\mathrm{RF}, k}\left(t-\left(k-\frac{1}{2}\right) \frac{\beta_{\mathrm{RF}}}{\omega_{\mathrm{RF}}}\right) \cdot p_{1}\left(t-\left(k-\frac{1}{2}\right) \frac{\beta_{\mathrm{LO}}}{\omega_{\mathrm{LO}}}\right)$

where $p_{1}(t)$ is a periodic waveform at the LO frequency $\omega_{\mathrm{LO}}$ as shown in Fig. 4. $p_{1}(t)$ represents the instantaneous current gain of the switching pair from drain of the current tail to the differential output terminal of the switching pair, and is expressed in terms of the instantaneous transconductances of the switch-pair transistors $g_{m 1}$ and $g_{m 2}$ [2]

$$
p_{1}(t)=\frac{g_{m 1}(t)-g_{m 2}(t)}{g_{m 1}(t)+g_{m 2}(t)} .
$$

The current gain $p_{1}(t)$ only contains the odd harmonics due to the differential architecture of each single-balanced cell. The drain current flowing through each branch of the switch pair arrives at the tap-point of the corresponding IF T-line, and is

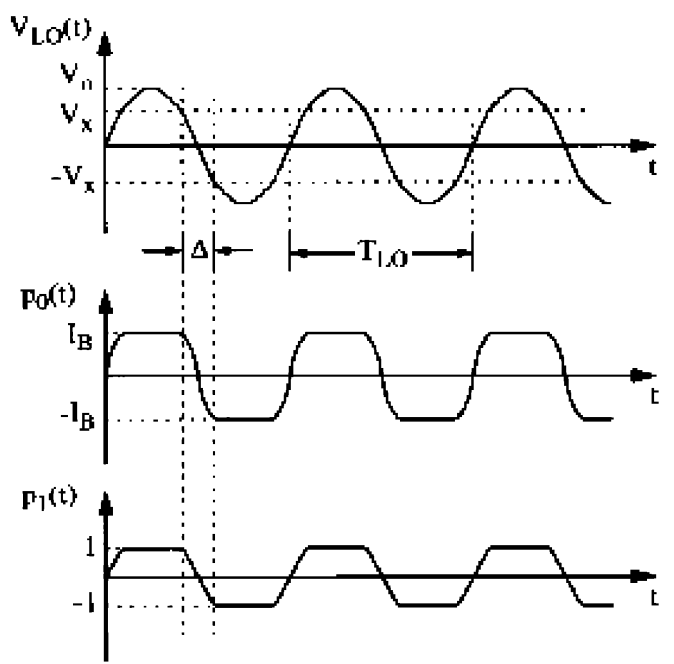

Fig. 4. Waveforms of $p_{1}(t)$ and $p_{0}(t)$ [2].

equally divided into two components; forward current component and reverse current component. Current components travel in opposite directions along the IF T-line with the forward component propagating toward the load and the reverse component propagating toward the input IF termination.

With a sufficiently large LO amplitude, the Fourier series coefficient of the fundamental frequency component of the instantaneous current gain of each single-balanced cell is approximated as [2]

$$
p_{11} \cong \frac{2}{\pi} \frac{\sin \left(f_{\mathrm{LO}} \Delta\right)}{f_{\mathrm{LO}} \Delta}
$$

where for a sinusoidal LO signal, $\pi f_{\mathrm{LO}} \Delta=\arcsin \left(V_{x} / V_{O}\right)$, and $V_{x}$ is the maximum differential input that guarantees the conducting transistor in the switching pair to remain in the saturation region for the entire switching interval [2], and $V_{O}$ is the maximum amplitude of the LO signal. The input LO voltage will, however, travel through the LO T-line before getting to the input terminals of each mixer cell. Both phase and amplitude of the LO signal are, therefore, changed while the LO signal is traveling down the LO T-line. As a result, $p_{11}$ becomes a function of the propagation constant of LO T-line. More precisely, the fundamental component of the current gain $p_{1}(t)$ for the $k$ th mixer cell is written as

$$
p_{11, k}=\frac{2}{\pi} \frac{V_{x} /\left(V_{o} e^{-\left(k-\frac{1}{2}\right) \alpha_{\mathrm{LO}}}\right)}{\arcsin \left(V_{x} /\left(V_{o} e^{-\left(k-\frac{1}{2}\right) \alpha_{\mathrm{LO}}}\right)\right)} e^{-j\left(k-\frac{1}{2}\right) \beta_{\mathrm{LO}}}
$$

where $\alpha_{\mathrm{LO}}$ and $\beta_{\mathrm{LO}}$ are the attenuation and phase constant of LO T-line, respectively. The attenuation constant of the LO $\mathrm{T}$-line is as follows [9]:

$$
\alpha_{\mathrm{LO}}=\frac{\left(\omega_{C} / \omega_{g \mathrm{LO}}\right) X_{\mathrm{LO}}^{2}}{\sqrt{1-\left[1-\left(\frac{\omega_{C}}{\omega_{g \mathrm{LO}}}\right)^{2}\right] X_{\mathrm{LO}}^{2}}}
$$

where $X_{\mathrm{LO}}=\omega_{\mathrm{LO}} / \omega_{C}$ is the normalized LO frequency, $\omega_{g \mathrm{LO}}=1 / r_{g \mathrm{LO}} C_{\mathrm{gs} \mathrm{LO}}$ is the gate cutoff frequency of the switching pair. Equation (7) indicates that the attenuation constant of LO T-line is a function of the LO frequency. For 


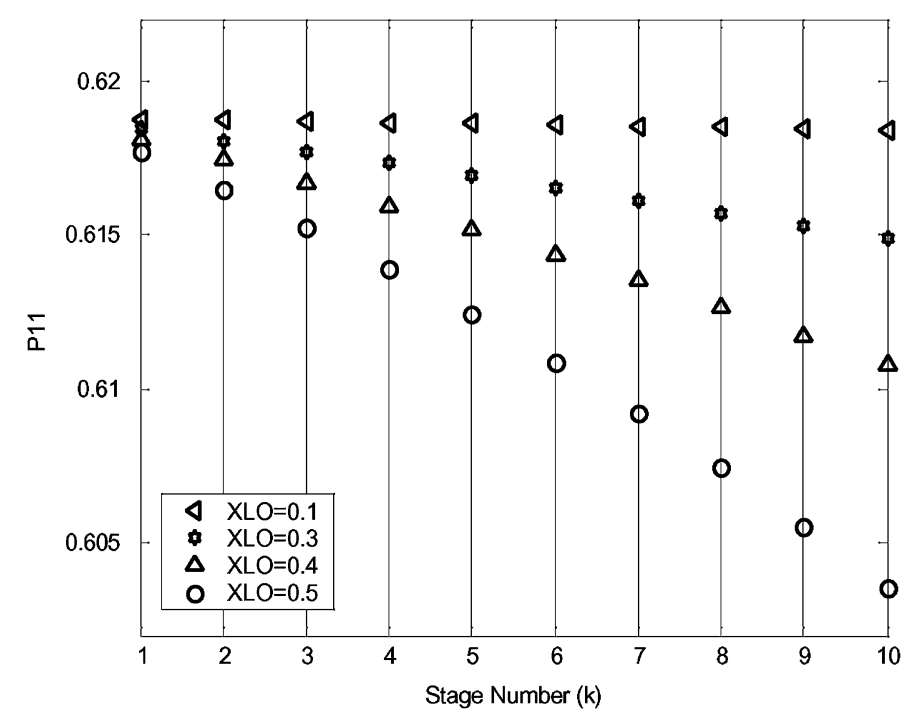

(a)

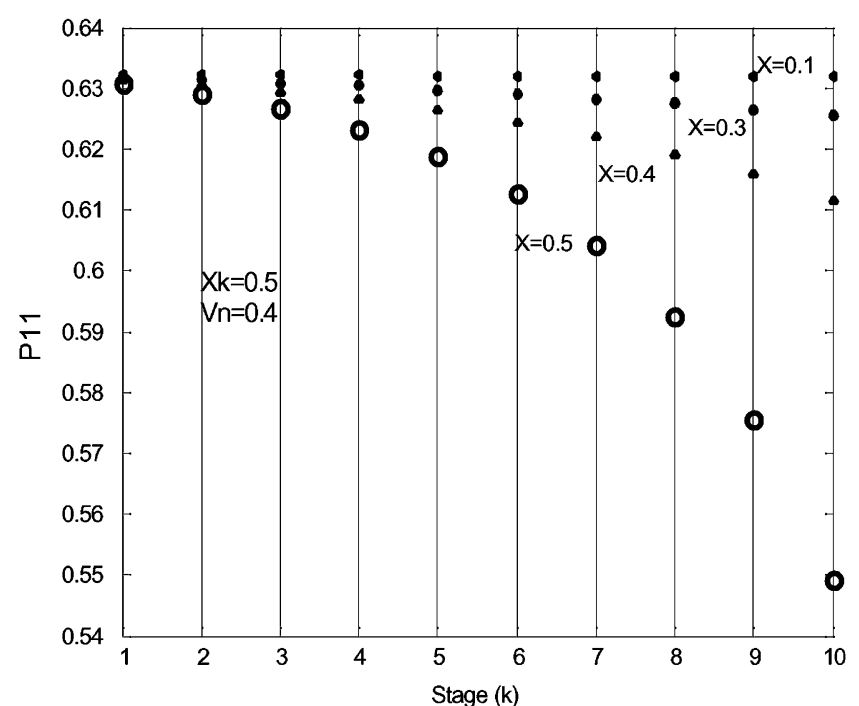

(b)

Fig. 5. Gain of the switching pair gain versus stage number for different $\mathrm{LO}$ frequencies. (a) $X_{k}=0.1$. (b) $X_{k}=0.5$.

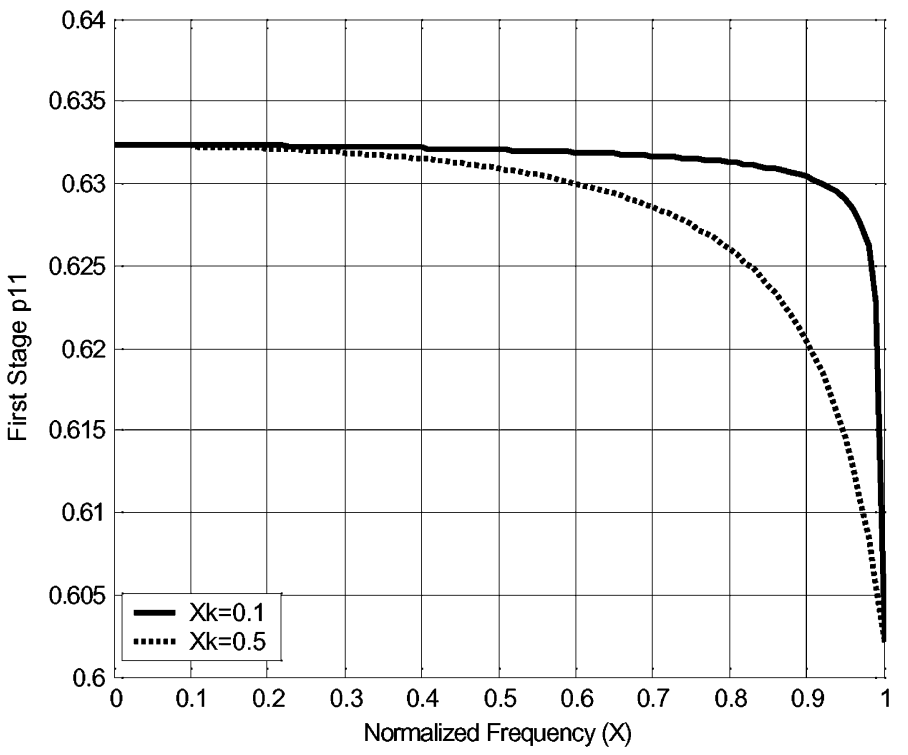

(a)

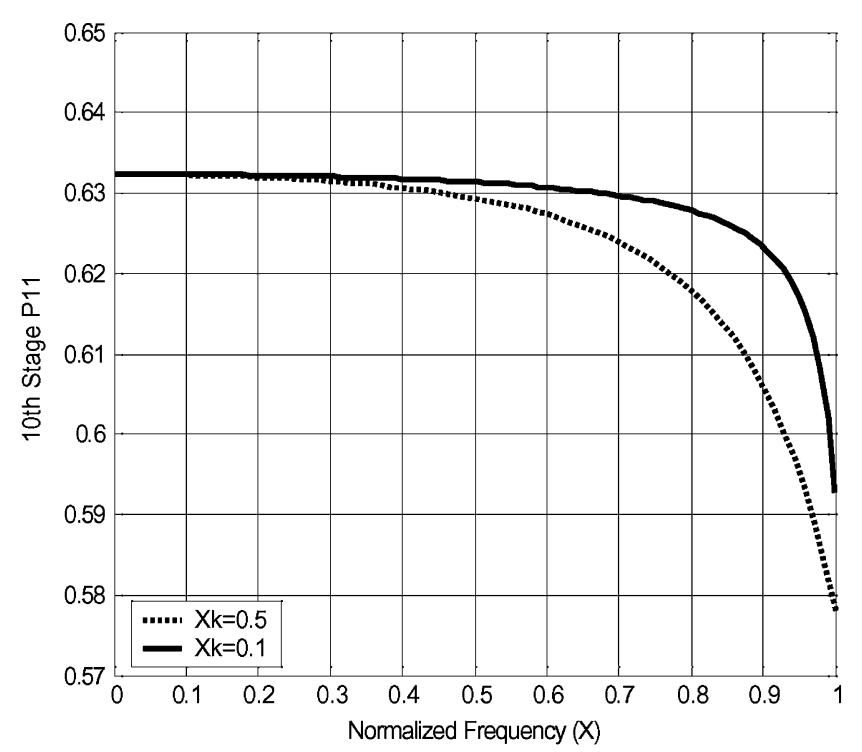

(b)

Fig. 6. $\quad p_{11}$ of (a) the first mixer cell and (b) the 10th mixer cell.

the UWB applications where LO frequency varies over a wide range of frequencies, one needs to examine the variation of $\alpha_{\mathrm{LO}}$ and its effect on the gain of the switching pair $p_{11, k}$. As will be shown using simulations, the gain of the switching pair is almost invariant for wide range of LO signal frequencies. This allows us to simplify the formulations.

Fig. 5(a) and (b) indicate variations of $p_{11, k}$ with respect to the stage number for two values of $X_{k}=\omega_{C} / \omega_{g}$ LO $\left(X_{k}=0.1\right.$ and 0.5 ). In this example the number of stages is assumed to be $n=10$. According to these figures, $p_{11}$ varies less than $8 \%$ from the first mixer cell to the last one, when the LO frequency varies between zero and $\omega_{C} / 2$, The maximum discrepancy in $p_{11, k}$ over this frequency range occurs when $\omega_{\mathrm{LO}}=0.5 \omega_{C}$. This observation implies that the gain of the switching pair is almost identical for different stages and for wide range of LO frequen- cies, despite the fact that the LO signal is attenuated along the LO T-line.

Shown in Fig. 5(a) and (b), $p_{11, k}$ monotonically decreases as $k$ increases. Therefore, any frequency-domain analysis of $\left|p_{11, k}\right|$ is carried out only for the first and the last mixer cell, guaranteeing that $\left|p_{11, k}\right|$ for $k=1, n$ provide an upper and lower bounds for the gains of intermediate mixer cells. Fig. 6(a) and (b) demonstrate the frequency behavior of $\left|p_{11, k}\right|$ for the first and the last mixer cells. As can be seen from these figures, the current gain of the switching pair for the last stage at $0.5 \omega_{C}$ decreases less than $0.3 \%$ from its low frequency value, whereas the current gain of the switching pair for the first stage at $0.5 \omega_{C}$ decreases less than $0.15 \%$ from its low frequency value.

Considering Figs. 5-6 it is inferred that the gain of switching pair is approximately identical for all mixer cells and constant 


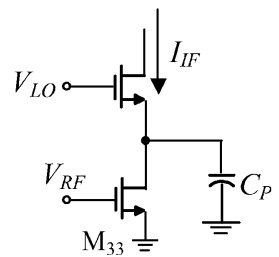

Fig. 7. Simplified circuit schematic of each mixer cell with a large amplitude LO input.

over a wide range of LO frequencies, particularly for the proposed mixer with four stages. Therefore, it is assumed that $\left|p_{11, k}\right|=p_{11}$.

Another important notion is that the parasitic capacitance $C_{p}$, seen at the common source node $\mathrm{P}$ in Fig. 1, significantly contributes on the high frequency roll-off of the conversion gain, because it is not absorbed by any existing $\mathrm{T}$-line in the proposed mixer. To include the effect of this capacitance in the analytical model, we postulate that during each half period of the LO input, only one of the transistors of the switch pair is on. This assumption is valid in practice, wherein the LO voltage has large amplitude, and $p_{1}(t)$ is approximately a square wave. As a consequence, each mixer cell is simplified to the circuit of Fig. 7.

The input-output transadmittance function from the RF input to the differential IF output is simply a first-order rational function of frequency

$$
\frac{I_{\mathrm{IF}}}{V_{\mathrm{RF}}}(j \omega)=g_{m \mathrm{RF}} p_{11} \frac{1}{1+\frac{j \omega C_{p}}{g_{m \mathrm{LO}}}}
$$

where $g_{m}$ LO is the instantaneous transconductance of the switching pair. Substituting (1)-(7) in the Fourier series of (3), and calculating the fundamental frequency component of the differential current leads to the following equation for the IF current traveling toward the load:

$$
I_{o}=\frac{1}{2} \frac{g_{m \mathrm{RF}} e^{-\frac{\gamma_{\mathrm{IF}}}{2}-j \tan ^{-1}\left(\frac{\omega_{\mathrm{RF}} C_{p}}{g_{m \mathrm{LO}}}\right)}}{\sqrt{1+\left(\frac{\omega_{\mathrm{RF}} C_{p}}{g_{m \mathrm{LO}}}\right)^{2}}} \sum_{k=1}^{n} p_{11, k}^{*} V_{i k} e^{-(n-k) \gamma_{\mathrm{IF}}}
$$

where * denotes the complex conjugate, as also specified in [11], and $\gamma_{\mathrm{IF}}$ is the propagation constant of the IF T-line.

Substituting (1) and (6) in (8), the total output current delivered to the load is written as (10)

$$
\begin{aligned}
& \left|I_{o}\right|=\frac{1}{2}\left|V_{\mathrm{RF}}\right| \\
& \times \frac{\left|p_{11}\right| g_{m \mathrm{RF}}}{\sqrt{1+\left(\frac{\omega_{\mathrm{RF}} C_{p}}{g_{m \mathrm{LO}}}\right)^{2}}} \frac{e^{-\frac{n}{2}\left(\alpha_{\mathrm{IF}}+\alpha_{\mathrm{RF}}\right)}}{\sqrt{1+\left(\frac{\omega_{\mathrm{RF}}}{\omega_{g \mathrm{RF}}}\right)^{2}}\left[1-\left(\frac{\omega_{\mathrm{RF}}}{\omega_{C}}\right)^{2}\right]} \\
& \times\left|\frac{\sinh \frac{n}{2}\left[\left(\alpha_{\mathrm{IF}}-\alpha_{\mathrm{RF}}\right)+j\left(\beta_{\mathrm{IF}}-\beta_{\mathrm{RF}}+\beta_{\mathrm{LO}}\right)\right]}{\sinh \frac{1}{2}\left[\left(\alpha_{\mathrm{IF}}-\alpha_{\mathrm{RF}}\right)+j\left(\beta_{\mathrm{IF}}-\beta_{\mathrm{RF}}+\beta_{\mathrm{LO}}\right)\right]}\right| .
\end{aligned}
$$

The attenuation of the RF T-line is obtained using a similar expression as that derived for the LO T-line (9), where, in this case, the frequency is normalized by $\omega_{\mathrm{RF}}$, and $X_{\mathrm{LO}}$ is replaced with $X_{\mathrm{RF}}$. The attenuation constant of the IF T-line is given by [9], [12]

$$
\alpha_{\mathrm{IF}}=\frac{\left(\omega_{d \mathrm{IF}} / \omega_{c}\right)}{\sqrt{1-X_{\mathrm{IF}}^{2}}}
$$

where $X_{\mathrm{IF}}$ is the normalized frequency and $\omega_{d \mathrm{IF}}=1 / r_{\mathrm{ds}} C_{\mathrm{ds}}$. The power delivered to the load, $P_{o}$, and the input power delivered to the amplifier, $P_{i}$, for the matched input and output terminations are given as follows:

$$
\begin{aligned}
P_{o} & =\frac{1}{2}\left|I_{o}\right|^{2} \operatorname{Re}\left[Z_{\mathrm{IF}}\right] \cong \frac{1}{2}\left|I_{o}\right|^{2} \sqrt{\frac{L_{\mathrm{IF}}}{C_{\mathrm{IF}}}\left(1-\left(\frac{\omega_{\mathrm{IF}}}{\omega_{C}}\right)^{2}\right)} \\
P_{i} & =\frac{\left|V_{\mathrm{RF}}\right|^{2}}{2\left|Z_{\mathrm{RF}}\right|^{2}} \operatorname{Re}\left[Z_{\mathrm{RF}}\right] \\
& \cong \frac{1}{2}\left|V_{\mathrm{RF}}\right|^{2} / \sqrt{\frac{L_{\mathrm{RF}}}{C_{\mathrm{RF}}}\left(1-\left(\frac{\omega_{\mathrm{RF}}}{\omega_{C}}\right)^{2}\right)}
\end{aligned}
$$

where $Z_{\mathrm{IF}}$ and $Z_{\mathrm{RF}}$ are the characteristics impedances of the IF and RF T-lines, respectively [9], [12]. Hence, the power gain of the distributed mixer is

$$
\begin{aligned}
G= & \frac{1}{4} \frac{\left|p_{11}\right|^{2} g_{m \mathrm{RF}}^{2}}{\left[1+\left(\frac{\omega_{\mathrm{RF}} C_{p}}{g_{m \mathrm{LO}}}\right)^{2}\right]} \\
& \times \frac{\sqrt{\frac{L_{\mathrm{IF}}}{C_{\mathrm{IF}}}\left(1-\left(\frac{\omega_{\mathrm{IF}}}{\omega_{C}}\right)^{2}\right)} \sqrt{\frac{L_{\mathrm{RF}}}{C_{\mathrm{RF}}}} e^{-n\left(\alpha_{\mathrm{IF}}+\alpha_{\mathrm{RF}}\right)}}{\left[1+\left(\frac{\omega_{\mathrm{RF}}}{\omega_{g \mathrm{RF}}}\right)^{2}\right]\left[1-\left(\frac{\omega_{\mathrm{RF}}}{\omega_{C}}\right)^{2}\right]^{3 / 2}} \\
& \times\left|\frac{\sinh ^{2} \frac{n}{2}\left[\left(\alpha_{\mathrm{IF}}-\alpha_{\mathrm{RF}}\right)+j\left(\beta_{\mathrm{IF}}-\beta_{\mathrm{RF}}+\beta_{\mathrm{LO}}\right)\right]}{\sinh ^{2} \frac{1}{2}\left[\left(\alpha_{\mathrm{IF}}-\alpha_{\mathrm{RF}}\right)+j\left(\beta_{\mathrm{IF}}-\beta_{\mathrm{RF}}+\beta_{\mathrm{LO}}\right)\right]}\right|
\end{aligned}
$$

From (13), the magnitude response of the conversion voltage gain of the single-balanced distributed mixer can be written as

$$
\begin{aligned}
A_{v}= & \frac{1}{4} \frac{\left|p_{11}\right| g_{m \mathrm{RF}}}{\sqrt{1+\left(\frac{\omega_{\mathrm{RF}} C_{p}}{g_{m \mathrm{LO}}}\right)^{2}}} \\
& \times \frac{\left[\left(\frac{L_{\mathrm{IF}}}{C_{\mathrm{IF}}}\right)\left(\frac{L_{\mathrm{RF}}}{C_{\mathrm{RF}}}\right)\left(1-\left(\frac{\omega_{\mathrm{IF}}}{\omega_{C}}\right)^{2}\right)\right]^{1 / 4} e^{-\frac{n}{2}\left(\alpha_{\mathrm{IF}}+\alpha_{\mathrm{RF}}\right)}}{\sqrt{1+\left(\frac{\omega_{\mathrm{RF}}}{\omega_{g \mathrm{RF}}}\right)^{2}}\left[1-\left(\frac{\omega_{\mathrm{RF}}}{\omega_{C}}\right)^{2}\right]^{3 / 4}} \\
& \times \mid \frac{\sinh \frac{n}{2}\left[\left(\alpha_{\mathrm{IF}}-\alpha_{\mathrm{RF}}\right)+j\left(\beta_{\mathrm{IF}}-\beta_{\mathrm{RF}}+\beta_{\mathrm{LO}}\right)\right]}{\sinh \frac{1}{2}\left[\left(\alpha_{\mathrm{IF}}-\alpha_{\mathrm{RF}}\right)+j\left(\beta_{\mathrm{IF}}-\beta_{\mathrm{RF}}+\beta_{\mathrm{LO}}\right)\right]} .
\end{aligned}
$$

The conversion gain $A_{v}$ in (14) has very high frequency poles at $\omega_{C}, \omega_{g \mathrm{RF}}$, and $g_{m \mathrm{LO}} / C_{p}$ for a varying RF frequency, which have a negligible impact on the frequency response of the conversion gain in the RF frequency range of interest. The fluctu- 


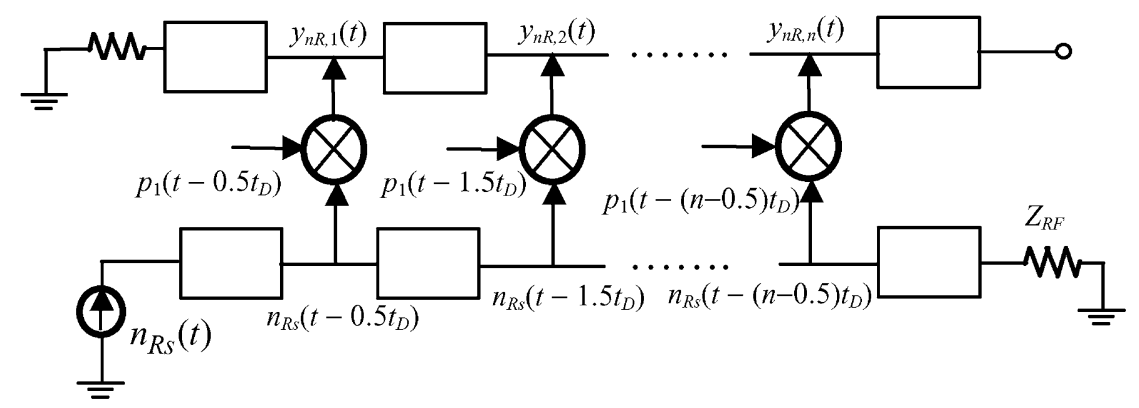

Fig. 8. Block diagram representation of the proposed mixer with the noise current $n_{R s}(t)$ due to the thermal noise resistance.

ations of the conversion gain over the RF frequency range are mostly due to the expression of

$$
\left|\frac{\sinh \frac{n}{2}\left[\left(\alpha_{\mathrm{IF}}-\alpha_{\mathrm{RF}}\right)+j\left(\beta_{\mathrm{IF}}-\beta_{\mathrm{RF}}+\beta_{\mathrm{LO}}\right)\right]}{\sinh \frac{1}{2}\left[\left(\alpha_{\mathrm{IF}}-\alpha_{\mathrm{RF}}\right)+j\left(\beta_{\mathrm{IF}}-\beta_{\mathrm{RF}}+\beta_{\mathrm{LO}}\right)\right]}\right| .
$$

To maximize the conversion gain, the argument of this term should be zero. This implies that the phase constant of the IF T-line must be

$$
\beta_{\mathrm{IF}}=\beta_{\mathrm{LO}}-\beta_{\mathrm{RF}}
$$

Equation (15) states the phase constant of the IF T-line should be equal to the difference between the phase constants of the RF and LO T-lines. In an ideal lossless artificial T-line, the phase constant is $\beta=2 \omega / \omega_{c}$. As a result, for the RF, LO, and IF $\mathrm{T}$-lines with identical cutoff frequencies, (15) becomes

$$
\omega_{\mathrm{IF}}=\left|\omega_{\mathrm{LO}}-\omega_{\mathrm{RF}}\right| .
$$

This implies that the proposed circuit topology of Fig. 1, always achieves its maximum conversion gain for a wide range of $\mathrm{RF}$ and $\mathrm{LO}$ frequencies.

It is clear from (7) and (15) that the attenuation of the IF and RF T-lines can be decreased by making $\omega_{\mathrm{IF}} / \omega_{C}$ and $\omega_{C} / \omega_{g} \mathrm{RF}$ small [12].

\section{NOISE ANALYSIS}

Generally speaking, the output signals of mixer cells in the $n$-stage distributed mixer of Fig. 1, after traveling toward the output terminal of the mixer, add together linearly at the output. Consequently, the output signal power is proportional to $n^{2}$. On the other hand, the output noise power contributed by each stage, linearly adds up with output noise powers contributed by other stages at the output of the mixer, assuming noise signals at the output of the mixer cells to be mutually independent. Hence, it is expected that the NF will be reduced by a factor of $n$ in the proposed distributed mixer compared to conventional single-stage mixers. However, such observation is over-simplistic and not accurate, partly because the existence of T-line resistive terminations introduces some additional thermal noise sources to the proposed distributed mixer architecture, thereby increasing the $\mathrm{NF}$ of the distributed mixer. However, as will be shown later, the contribution of these thermal noise sources on the overall $\mathrm{NF}$ is also divided by a factor of $n$. Moreover, the existence of correlated terms increases the NF, as will be explained in more details.

The dominant intrinsic noise sources in the proposed distributed mixer are:

1) thermal noise from the input source impedance $\left(R_{S}\right)$;

2) thermal noise from the RF, LO, and IF terminations;

3) noise sources associated with each of MOS transistor including the thermal noise from $r_{g}$ (polysilicon gate resistance), and channel noise.

As an assumption, the impact of the upconverted frequency components of the flicker noise on the overall NF of the distributed mixer is neglected.

To investigate the noise performance of the proposed distributed mixer, the noise contribution of each mixer cell is first studied, while ignoring the effects of parasitic capacitances. Each mixer cell contains two signal paths: 1) the RF input and 2) The LO input. The noise analysis of each mixer cell is thus decomposed into two parts.

\section{A. Noise From the Driver Stage (RF Stage)}

1) Noise From the Source Impedance and RF T-Line Termination: Consider the thermal noise current component $n_{R s}(t)$ due to the input source impedance, as shown in Fig. 8. Each mixer in Fig. 8 is shown using an ideal multiplier with one input coming from the RF line and other input representing the instantaneous current gain.

The noise source $n_{R s}(t)$ is considered to be a wide-sense stationary (WSS) process with power spectral density (PSD) $S_{n_{R s}}(f)$ whose value is $g_{m \mathrm{RF}}^{2}\left(4 k T R_{S}\right)$, where $k$ is Boltzman's constant, and $T$ is the absolute temperature. The noise current $n_{R s}(t)$ also travels down the RF T-line and contributes noise at the input of each mixer cell. The noise component at the output of the $k$ th mixer cell due to $n_{R s}(t)$ is

$$
\begin{aligned}
y_{n R, k}(t)=\frac{1}{2} n_{R s}\left(t-\left(k-\frac{1}{2}\right) t_{\mathrm{RF}}\right) & \\
\cdot & p_{1}\left(t-\left(k-\frac{1}{2}\right) t_{\mathrm{LO}}\right)
\end{aligned}
$$

where $t_{\mathrm{RF}}$ and $t_{\mathrm{LO}}$ are delays due to each segment of RF and LO T-lines, respectively. Furthermore, $t_{\mathrm{LO}}=t_{\mathrm{RF}}=t_{\mathrm{IF}}=$ $t_{D}$, because in an artificial transmission line the delay caused by each delay line segment is $t_{\text {Delay }}=\sqrt{L C}$, and $\sqrt{L C}$ is designed to be identical for RF, LO, and IF artificial T-lines. The noise current appearing at the output of the kth cell experiences 
a $(n-k+1 / 2) t_{D}$ unit delay to reach at the load termination, hence, becomes (for $k=1,2, \ldots, n$ )

$$
y_{n_{R s}, k}^{o}(t)=\frac{1}{4} n_{R s}\left(t-n t_{D}\right) \cdot p_{1}\left(t-n t_{D}\right) .
$$

The thermal noise due to the source resistance, and its replica components traveling through $n$ individual mixer cells are correlated, because they have the same noise source. The additional $1 / 2$ factor comes from the fact that at each tap-point of the IF T-line, the noise evenly splits into two correlated terms. Recall that $p_{1}(t)$ is a periodic signal, which makes $y_{n_{R s}, k}^{o}(t)$ a cyclostationary process [18].

Cyclostationary process further complicates the noise analysis, because the PSD of $y_{n_{R}, k}^{o}(t)$ (as a cyclostationay process) becomes a function of both time and frequency. However, the time-average of a cyclostationary process is a WSS process [18]. Therefore, the time-average of $y_{n_{R}, k}^{o}(t)$ is taken into consideration. The autocorrelation of the total noise current at the load terminal contributed by the thermal noise due to the source resistance $n_{R s}(t)$ is

$$
\overline{\left\langle y_{n_{R s}}^{o}\right\rangle^{2}}=\sum_{k=1}^{n} \overline{\left\langle y_{n_{R s}, k}^{o}\right\rangle^{2}}+\sum_{i=1}^{n} \sum_{k=1}^{n} \overline{\left\langle y_{n_{R s}, k}^{o}\right\rangle \cdot\left\langle y_{n_{R s}, i}^{o}\right\rangle}
$$

where $\langle x(t)\rangle$ denotes the time average of the signal $x(t)$. The first additive term in (17) represents the summation of the average noise powers of replica components of the thermal noise due to the source resistance. The second additive term represents the summation of the crosscorrelations of any two replica components of the thermal noise due to the source resistance that propagate through any two distinct mixer paths toward the output terminal. Interestingly, the thermal noise $n_{R s}(t)$ and its all the replica components traveling through the mixer cells experience the same propagation delay from the RF source terminal to the IF output terminal, which means: $\left\langle y_{n_{R s}, i}^{o}\right\rangle=\left\langle y_{n_{R s}, j}^{o}\right\rangle$ for $i \neq j$. Equation (17) thus becomes

$$
\overline{\left\langle y_{n_{R s}}^{o}\right\rangle^{2}}=\frac{n^{2}}{16}\left\langle p_{1}^{2}(t)\right\rangle \overline{n_{R s}^{2}}
$$

where $\left\langle p_{1}^{2}(t)\right\rangle$ is the average power of $p_{1}(t)$, which can be estimated as [2]

$$
\left\langle p_{1}^{2}(t)\right\rangle=E_{p} \cong 1-\frac{4}{3}\left(\Delta f_{\mathrm{LO}}\right)
$$

where for a sinusoidal LO waveform, $\Delta f_{\mathrm{LO}}$ is obtained using (5). The time-average PSD of (18) is as follows:

$$
\overline{\left\langle y_{n_{R} s}^{o}\right\rangle^{2}}=\frac{n^{2}}{16} E_{p} g_{m \mathrm{RF}}^{2}\left(4 k T R_{S}\right) .
$$

The noise contribution of the RF $\mathrm{T}$-line termination $\left(Z_{\mathrm{RF}}\right), n_{Z \mathrm{RF}}(t)$, at the output terminal is similarly expressed as the summation of the average noise power of $n_{Z \mathrm{RF}}(t)$ and all its replica components, with all cross correlation terms becoming zero, because the noise components experience different propagation delay to the IF output terminal [see (21)]

$$
\overline{\left\langle y_{n_{Z \mathrm{RF}}}^{o}\right\rangle^{2}}=\sum_{k=1}^{n} \overline{\left\langle y_{n_{Z \mathrm{RF}}, k}^{o}\right\rangle^{2}}=\frac{n}{16} E_{p} g_{m \mathrm{RF}}^{2}\left(4 k T \operatorname{Re}\left[Z_{\mathrm{RF}}\right]\right) \text {. }
$$

2) Channel Thermal Noise of the Tail Current Transistor: Each mixer cell includes a current tail whose ac current is controlled by the RF input voltage. The current tail transistor within each mixer cell exhibits a channel current noise, which flows through the switching pair, and then propagates though the IF T-line. The output noise currents of the mixer cells due to the channel current noise of the constituent current tails are mutually uncorrelated. As a consequence, the overall noise power at the IF output termination due to the channel current noise of current tail transistors is obtained by simply adding up the PSD of noise currents of individual mixer cells.

The time-average PSD of the total output current noise contributed by the channel current noise of all constituent tail currents is obtained as follows

$$
\overline{\left\langle y_{n \mathrm{Id}}^{o}\right\rangle^{2}}=\frac{n}{4} E_{p}\left(4 k T \gamma_{3} g_{m \mathrm{RF}}\right)
$$

where $\gamma_{3}$ is $2 / 3$ for long channel devices, and higher for short channel devices [19].

3) Noise From Polysilicon Gate Resistance of the Driver Stage $\left(r_{g \mathrm{RF}}\right)$ : In contrast to the noise contribution of the source resistance $R_{s}$, the noise due to the polysilicon gate resistance $r_{g}$ of each driver stage contributes noise at the output through two major paths, forward paths and backward paths, as depicted in Fig. 9. With identical RF, IF, and LO T-lines in the proposed circuit of Fig. 1, the noise components traveling through the forward paths all experience the same delay. For the $k$ th mixer cell, $(n-k+1)$ correlated noise components due to the thermal noise of the polysilicon gate resistance will superimpose at the output. The time-average PSD of the forward traveling noise due to $r_{g \mathrm{RF}}$ of the $k$ th cell becomes

$$
\overline{\left\langle y_{n_{r g}, k}^{F}\right\rangle^{2}}=\frac{1}{4}(n-k+1)^{2} E_{p} g_{m \mathrm{RF}}\left(4 k T r_{g \mathrm{RF}}\right)
$$

where superscript $F$ denotes the forward path. As for the backward path, $(k-1)$ noise components arrive at the output with different propagation delay, hence becoming uncorrelated. As a consequence, the time-average PSD of these uncorrelated noise components will directly add up at the output, i.e.,

$$
\overline{\left\langle y_{n_{r g}, k}^{B}\right\rangle^{2}}=\frac{1}{4}(k-1) E_{p} g_{m \mathrm{RF}}\left(4 k T r_{g \mathrm{RF}}\right) .
$$

The overall output noise PSD of the $k$ th cell is obtained as the summation of the PSDs of forward and backward components, because they are uncorrelated. Moreover, the overall output noise PSD of all the other cells are uncorrelated. Therefore, the overall output noise PSD due to the thermal noise of all $r_{g R F, k}(k=1, \ldots, n)$ becomes

$$
\begin{aligned}
& \overline{\left\langle y_{n_{r g}, \mathrm{RF}}^{o}\right\rangle^{2}} \\
& \quad=\frac{1}{4} \sum_{k=1}^{n}\left[(n-k+1)^{2}+(k-1)\right] E_{p} g_{m \mathrm{RF}}\left(4 k T r_{g \mathrm{RF}}\right) \\
& \quad=\frac{n\left(n^{2}+3 n-1\right)}{12} E_{p} g_{m \mathrm{RF}}\left(4 k T r_{g \mathrm{RF}}\right) .
\end{aligned}
$$

As an important observation, the thermal noise sources of polysilicon gate resistances of first stages have dominant noise contribution at the output. Therefore, cares must be taken into 


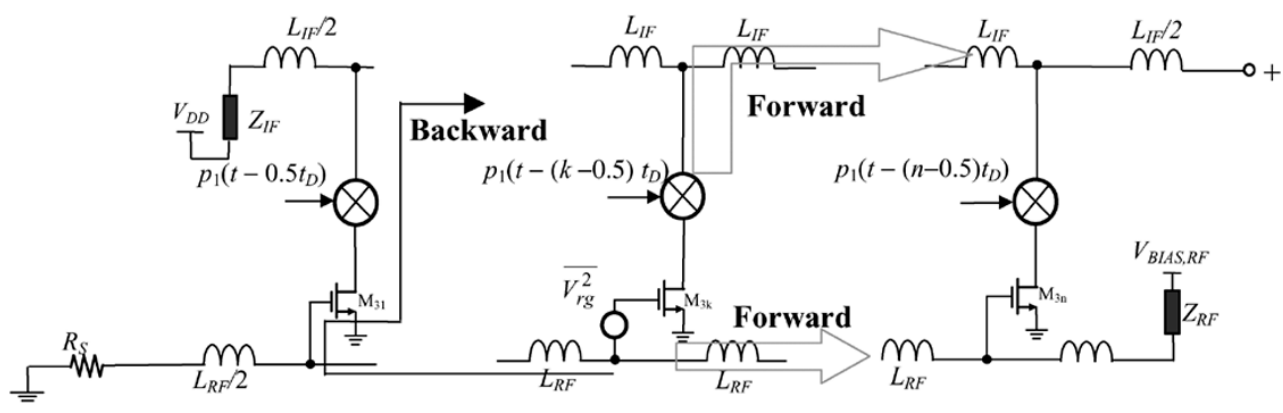

Fig. 9. Simplified circuit model for noise contribution of $r_{g \text { RF }}$ of the $k$ th mixer cell.

account to minimize the polysilicon gate resistance of the first stages within the proposed mixer circuit.

\section{B. Noise From LO Stage}

1) Thermal Noise From Drain Current: During the time interval within each LO period when only one of the switching pair transistors is conducting, the output current is determined by $I_{\mathrm{RF}}$ that is generated by the driver stage. The mixer cell is then simplified to the circuit shown in Fig. 7. The switchpair transistor contributes negligible noise at the output of each mixer cell. Therefore, the output noise is dominated by the noise from driver stage. During the interval when both switching pair transistors are conducting, they contribute noise to the output and the corresponding time-average PSD of the output noise of the $k$ th mixer stage, traveling toward the load is [2]

$$
\overline{\left\langle y_{n_{\mathrm{LO}}, k}^{o}\right\rangle^{2}}=\frac{1}{4} 8 k T \gamma_{12}\left\langle G\left(t-\left(k-\frac{1}{2}\right) t_{D}\right)\right\rangle
$$

where

$$
G(t)=2 \frac{g_{m \mathrm{LO}_{1}}(t) \cdot g_{m \mathrm{LO}_{2}}(t)}{g_{m \mathrm{LO}_{1}}(t)+g_{m \mathrm{LO}_{2}}(t)}
$$

is the instantaneous transconductance of the differential pair, $\langle G(t)\rangle$ is the time average of $G(t)$, and $g_{m \mathrm{LO} 1,2}$ are the transconductances of each device in the switching pair. The estimated value for $\langle G(t)\rangle$ is calculated by [2], and simply equals to $2 I_{B} / \pi V_{O}$, where $I_{B}$ is the bias current of the tail current. Because of the periodic nature of $G(t)$, its time average is invariant to the propagation delay $t_{D}$ appeared in (25), i.e.,

$$
\overline{G\left(t-\left(k-\frac{1}{2}\right) t_{D}\right)}=\overline{G(t)} \text {. }
$$

Once again, the drain current noise sources from each mixer cell are uncorrelated. Therefore, the total output PSD of the current noise is $n$ times that of each individual mixer cell

$$
\overline{\left\langle y_{n \mathrm{LO}}^{o}\right\rangle^{2}}=\frac{n}{4}\left(8 k T \gamma_{12} \overline{G(t)}\right) \text {. }
$$

2) Noise Contribution of $r_{g}$ LO and LO Terminations: Assuming that the $k$ th mixer cell exhibits a noise component $n_{r g \mathrm{LO}}(t)$ due to the polysilicon gate resistance of switching pair transistors, the noise contributed by $n_{r g \text { LO }}(t)$ at the output of the $k$ th mixer cell is given by

$$
y_{n r g, \mathrm{LO}}(t)=n_{r g \mathrm{LO}}(t) G\left(t-\left(k-\frac{1}{2}\right) t_{D}\right)
$$

where $G(t)$ is defined by (27), and is a periodic function. Therefore, $y_{n r g, \mathrm{LO}}$ becomes a cyclostationary process.

The overall noise contribution of $r_{g}$ LO is similar to (27), discussed in Section III. Consequently, the overall output noise PSD due to thermal noise of all $r_{g \text { LO }, k}(k=1, \ldots, n)$ becomes

$$
\begin{aligned}
\overline{\left\langle y_{n r g, \mathrm{LO}}^{o}\right\rangle^{2}} & =\frac{1}{4} \sum_{k=1}^{n}\left[(n-k+1)^{2}+(k-1)\right] E_{G}\left(4 k T r_{g \mathrm{LO}}\right) \\
& =\frac{n\left(n^{2}+3 n-1\right)}{12} E_{G}\left(4 k T\left(2 r_{g \mathrm{LO}}\right)\right)
\end{aligned}
$$

where $E_{G}$ denotes the average power of $G(t) . E_{G}$ is expressed as follows [2]:

$$
E_{G}=4.64 \frac{K_{1}^{1 / 2} I_{B}^{3 / 2}}{\lambda T_{\mathrm{LO}}}
$$

where parameter $K_{1}$ depends on technology and the size of the device and is proportional to the channel width [2], $I_{B}$ is driver bias current, $T_{\mathrm{LO}}$ is LO signal period, and $\lambda$ is the zero crossing slope of LO signal. For a sinusoidal LO signal with amplitude $V_{o}, \lambda=2 \pi V_{o} / T_{\mathrm{LO}}$.

Each switching pair is comprised of two NMOS devices, hence a multiplying factor of two for the thermal noise contribution of each cell, as also seen in (30). The noise contribution of the LO T-line termination $\left(Z_{\mathrm{LO}}\right), n_{Z \mathrm{LO}}(t)$, at the output terminal of the proposed mixer obeys a similar mechanism discussed in Section A1 regarding the noise contribution of the RF T-line termination, with a minor difference; $n_{Z \mathrm{LO}}(t)$ at the gate terminal of the switching pair device experiences the instantaneous transconductance of the differential pair $G(t)$ to arrive at the IF T-line. Therefore, the overall output noise PSD due to the thermal noise of $Z_{\mathrm{LO}}$ is obtained as

$$
\overline{\left\langle y_{n Z \mathrm{LO}}^{o}\right\rangle^{2}}=\sum_{k=1}^{n} \overline{\left\langle y_{n_{Z \mathrm{LO}}, k}^{o}\right\rangle^{2}}=\frac{n}{16} E_{G}\left(4 k T\left(2 \operatorname{Re}\left[Z_{\mathrm{LO}}\right]\right)\right) \text {. }
$$

\section{Noise Contribution of IF Termination}

Noise sources due to the IF terminations $Z_{\text {IF }}$ propagate along the IF T-lines and appear at the IF output terminals with some delay which does not affect the noise calculations, because this noise is a WSS process. Hence, the PSD of the output noise current due to the thermal noise of $Z_{\mathrm{IF}}$ is written as

$$
\overline{y_{n Z \mathrm{IF}}^{o^{2}}}=\frac{1}{2}\left(\frac{4 k T}{\operatorname{Re}\left[Z_{\mathrm{IF}}\right]}\right) \text {. }
$$




\section{Mixer NF}

So far, we calculated the noise contributions of various noise sources at the output of the proposed distributed mixer. Results of these calculations are directly incorporated in the definition of the NF [10]

$$
\mathrm{NF}=\frac{\overline{I_{\text {nout }}^{2}}}{A_{I}^{2}} \frac{1}{4 K T / R_{S}}
$$

where

$$
A_{I}=\frac{1}{4} \operatorname{cng}_{m \mathrm{RF}} R_{S}
$$

is the current gain of the $n$-stage distributed mixer, and $c=$ $\left|p_{11}\right|$ is the gain of switching pair at the IF frequency, which is defined in (7). $\overline{I_{n \text { out }}^{2}}$ is the overall PSD of the output noise current whose value is determined as the summation of noise expressions in (19), (21), (22), (25), (28), (30), (32), and (33). The single-sideband (SSB) NF of the distributed single balanced mixer is thus expressed as

$$
\begin{aligned}
(\mathrm{NF})_{\mathrm{SSB}}= & \frac{E_{P}}{c^{2}}+\frac{E_{P}}{n c^{2}} \frac{\operatorname{Re}\left[Z_{\mathrm{RF}}\right]}{R_{S}}+\frac{4 E_{P}}{n c^{2} g_{m \mathrm{RF}}} \frac{\gamma_{3}}{R_{S}} \\
& +\frac{4 E_{P}\left(n^{2}+3 n-1\right)}{3 n c^{2}} \frac{r_{g \mathrm{RF}}}{R_{S}} \\
& +\frac{8}{n c^{2} g_{m \mathrm{RF}}^{2}} \frac{\gamma_{12} \bar{G}}{R_{S}}+\frac{4 E_{G}\left(n^{2}+3 n-1\right)}{3 n c^{2} g_{m \mathrm{RF}}^{2}} \frac{2 r_{g \mathrm{LO}}}{R_{S}} \\
& +\frac{E_{G}}{n c^{2} g_{m \mathrm{RF}}^{2}} \frac{2 \mathrm{Re}\left[Z_{\mathrm{LO}}\right]}{R_{S}}+\frac{1}{n^{2} c^{2} g_{m \mathrm{RF}}^{2}} \frac{2 / \operatorname{Re}\left[Z_{\mathrm{IF}}\right]}{R_{S}}
\end{aligned}
$$

Comparing to the NF of a single balanced mixer obtained in [2], there exist some additional terms in NF related to T-line terminations. From (36) it is seen that the noise contributions due to the RF and LO terminations are reduced by a factor of $n$, whereas those due to the IF terminations are reduced by a factor of $n^{2}$. Although the noise contributions due to the polysilicon gate resistances are increased by a factor of $n$, they are insignificant in the overall NF, because $r_{g \mathrm{LO}}, r_{g \mathrm{RF}} \ll R_{S}$. Besides, comparing (36) with the NF of the single-balance mixer obtained in [2] indicates that all the other terms in the NF expression are divided by $n$.

Of particular interest is to calculate the optimum number of stages to achieve the minimum NF. To calculate a closed form expression for the number of stages, we postulate that the last term in (36) is negligible for large $n$. The optimum number of

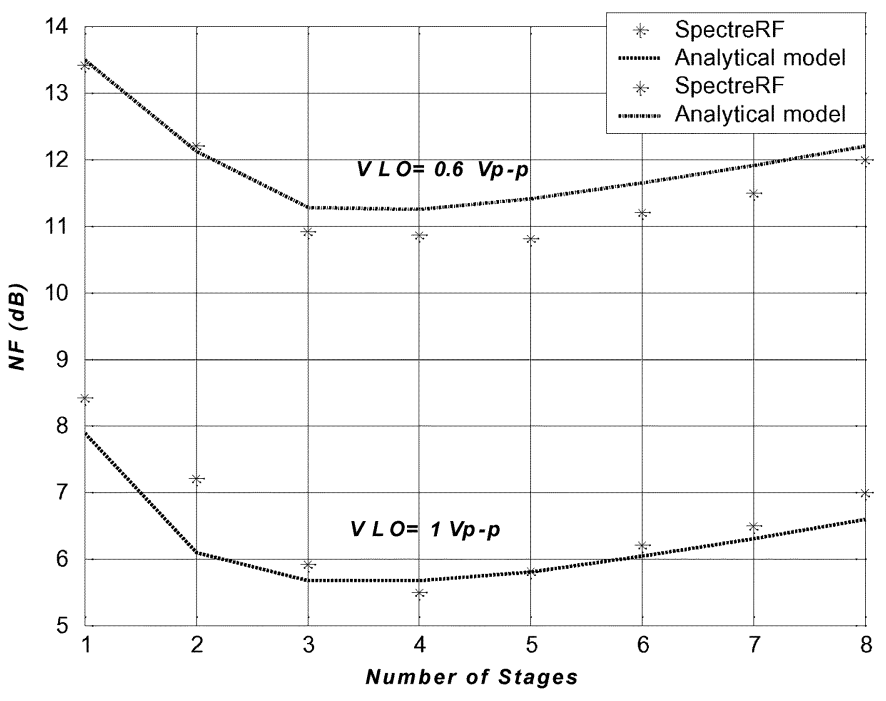

Fig. 10. Mixer NF for different number of stages.

stages $n_{\mathrm{opt}}$ and the minimum NF, NF $\mathrm{min}_{\text {in }}$, are calculated in (37) and (38), shown at the bottom of the page.

As the first step toward the operational verification of the proposed mixer circuit, the circuit has been designed in a $0.18-\mu \mathrm{m}$ standard CMOS process, and simulated using Spectre-RF. The simulation is carried out to verify the accuracy of the analytical model in (36)-(38). Depicted in Fig. 10 is the result of the comparison between the Spectre-RF simulation and the analytical models. As predicted by (37)-(38) and verified by the simulations, there is an optimum number of stages, which results in minimum NF. Clearly, the optimum number of stages stage depends on the circuit parameters such as gate resistance, transconductance of the RF tail current transistor, and also the voltage swing at the LO port [see (37)]. For lower voltage swings at the LO port, the differential pair transistors will no longer act like voltage-dependent switches, therefore, the mixer gain will decrease and the NF will increase.

\section{Measurement Results}

A two-stage distributed mixer was designed and fabricated in $0.18-\mu \mathrm{m}$ CMOS provide by the Jazz sbc18 process. The die photo of the proposed circuit is depicted in Fig. 11(a). The die area is $1.4 \times 1.16 \mathrm{~mm}^{2}$. The die was mounted on a 4 metal-layer high-frequency board, and directly wire-bonded. The board is demonstrated in Fig. 11(b). The microstrip lines directly connected the wire-bonded pads to the connectors. A 1.8-V supply

$$
\begin{aligned}
n_{\mathrm{opt}}= & \sqrt{\frac{E_{P}\left(\operatorname{Re}\left[Z_{\mathrm{RF}}\right]+\frac{4 \gamma_{3}}{g_{m \mathrm{RF}}}-\frac{4 r_{g \mathrm{RF}}}{3}\right)+\frac{8 \gamma_{12} \bar{G}}{g_{m \mathrm{RF}}^{2}}+E_{G}\left(\frac{2 \mathrm{Re}\left[Z_{\mathrm{LO}}\right]}{g_{m \mathrm{RF}}^{2}}-\frac{4}{3} \frac{2 r_{g \mathrm{LO}}}{g_{m \mathrm{RF}}^{2}}\right)}{3} r_{g \mathrm{RF}}+\frac{4 E_{G}}{3 g_{m \mathrm{RF}}^{2}} 2 r_{g \mathrm{LO}}} \\
\mathrm{NF}_{\min }= & \frac{E_{P}}{c^{2}}+\frac{4 E_{G}}{c^{2} g_{m \mathrm{RF}}^{2}} \frac{2 r_{g \mathrm{LO}}}{R_{S}}+\frac{4 E_{P}}{3 c^{2}} \frac{r_{g \mathrm{RF}}}{R_{S}}+\frac{2}{c^{2} R_{S}}\left[E_{P}\left(\operatorname{Re}\left[Z_{\mathrm{RF}}\right]+\frac{4 \gamma_{3}}{g_{m \mathrm{RF}}}-\frac{4 r_{g \mathrm{RF}}}{3}\right)\right. \\
& \left.+\frac{8 \gamma_{12} \bar{G}}{g_{m \mathrm{RF}}^{2}}+E_{G}\left(\frac{2 \mathrm{Re}\left[Z_{\mathrm{LO}}\right]}{g_{m \mathrm{RF}}^{2}}-\frac{4}{3} \frac{2 r_{g \mathrm{LO}}}{g_{m \mathrm{RF}}^{2}}\right)\right]^{1 / 2} \times\left(\frac{4 E_{P}}{3} r_{g \mathrm{RF}}+\frac{4 E_{G}}{3 g_{m \mathrm{RF}}^{2}} 2 r_{g \mathrm{LO}}\right)^{1 / 2}
\end{aligned}
$$


(a)

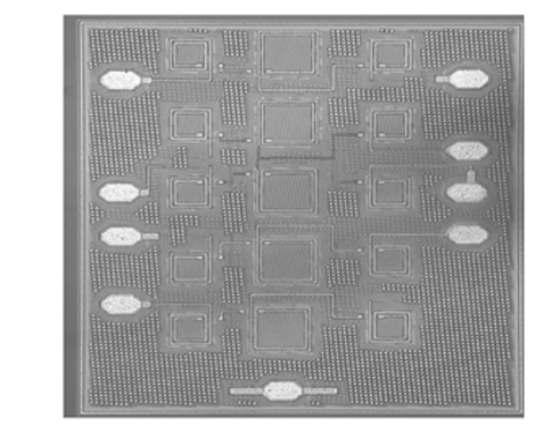

(b)

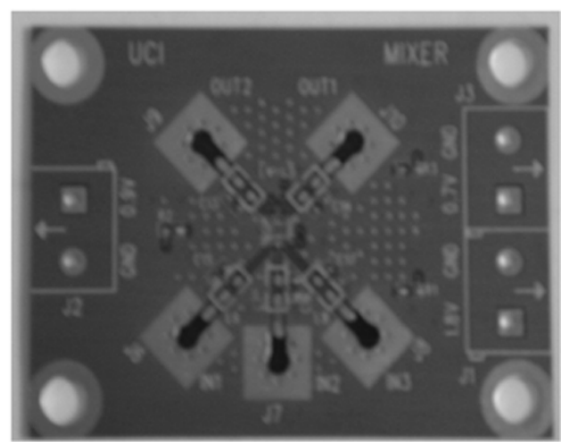

Fig. 11. Die photo of the distributed mixer. (a) Initial die photo. (b) Test board with the die mounted on it.

voltage was used in the design. The $1.8-\mathrm{V}$ power supply drew $6 \mathrm{~mA}$ of dc current.

In the first experiment, the conversion gain of the proposed mixer was measured for an IF frequency of $528 \mathrm{MHz}$. As recommended by 802.15.3a WPAN standard, the bandwidth of each sub-band in the multi-band OFDM UWB transceiver is $528 \mathrm{MHz}$. Therefore, we chose $528 \mathrm{MHz}$ as the IF frequency to test the performance of the mixer circuit. The LO and RF signal amplitudes were set to be 9 and $-3 \mathrm{dBm}$, respectively.

An HP 11667c power splitter with 6-dB of power-loss has been employed to produce the differential input for the LO signal. One of the IF outputs was terminated to the $50 \Omega$ load, and the other one was connected to the spectrum analyzer Agilent 8565 . We measured the single ended IF output, and used 6-dB correction factor to account for the 6-dB gain increase of the differential conversion gain. Fig. 12 shows the conversion gain with respect to the RF frequency. As shown in this figure, the conversion gain exhibited a $3-\mathrm{dB}$ variation across the $3-8.72-\mathrm{GHz}$ input frequency. The average conversion gain was around $3.0 \mathrm{~dB}$.

Fig. 13(a) demonstrates the output of the spectrum analyzer for the RF frequency of $8.712 \mathrm{GHz}$. The RF input power was set to $-27 \mathrm{dBm}$. Fig. 13(b) shows the result of the two-tone test for the mixer at the RF frequency of $5.016 \mathrm{GHz}$, and the LO frequency of $4.488 \mathrm{GHz}$. As indicated in Fig. 13(b), the proposed mixer circuit exhibited an extrapolated IIP 3 of $5.0 \mathrm{dBm}$. For this measurement, two RF input one at $5.013 \mathrm{GHz}$ and the other one at $5.019 \mathrm{GHz}$ were fed to the input RF port. Fig. 14 demonstrates the output of the spectrum analyzer for RF input power of $-15 \mathrm{dBm}$ and RF frequencies of 5.013 and $5.019 \mathrm{GHz}$.

The last experiment includes the s-parameter measurement to find out about the matching performance of the proposed mixer. The Agilent E5071A network analyzer with a maximum frequency bandwidth of $8.5 \mathrm{GHz}$ has been used for measuring the

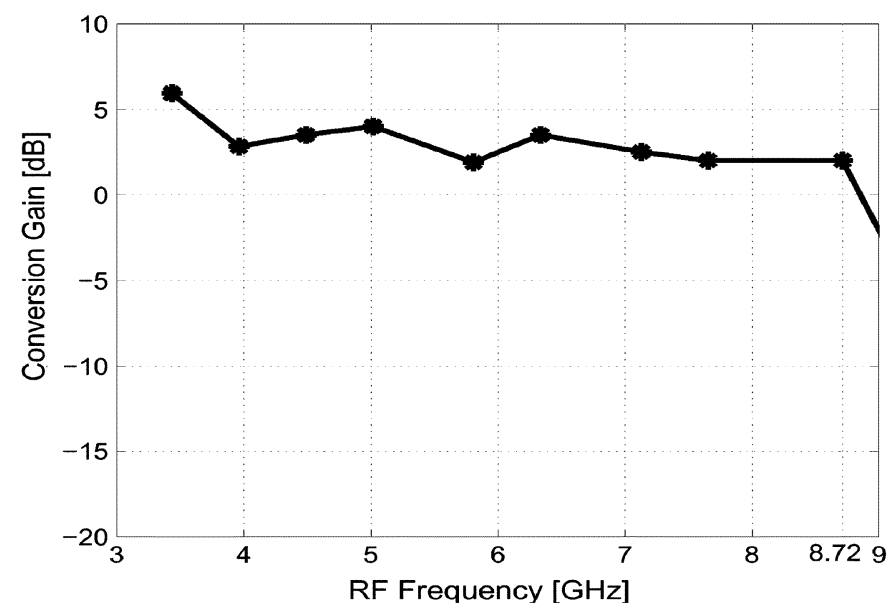

Fig. 12. Conversion gain versus RF.

(a)

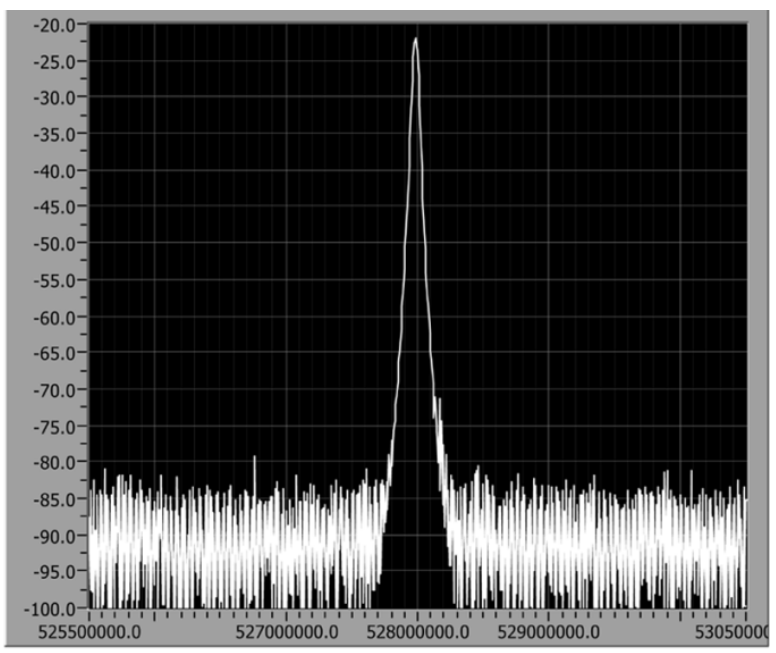

(b)

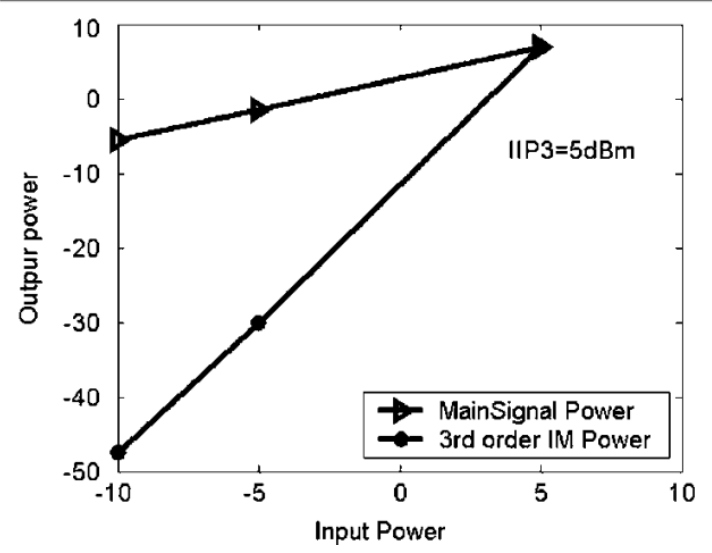

Fig. 13. (a) Output of the spectrum analyzer for the RF frequency of $8.712 \mathrm{GHz}$ and $\mathrm{RF}$ power of $-27 \mathrm{dBm}$. (b) Measured two-tone test.

input reflection coefficient, $\mathrm{s}_{11}$. All other output terminals were terminated to $50-\Omega$ line impedance. The measurement result of the magnitude response of $s_{11}$ is shown in Fig. 15. As also indicated in this figure, $s_{11}$ exhibited a good flat response of $-10 \mathrm{~dB}$ over the entire 3-8.5 GHz. The proposed mixer circuit also exhibited very good sensitivity to the LO variation. Our measurements show that if the LO signal varies from 9 to $20 \mathrm{dBm}$, the conversion gain will vary only by $1.5 \mathrm{~dB}$. 


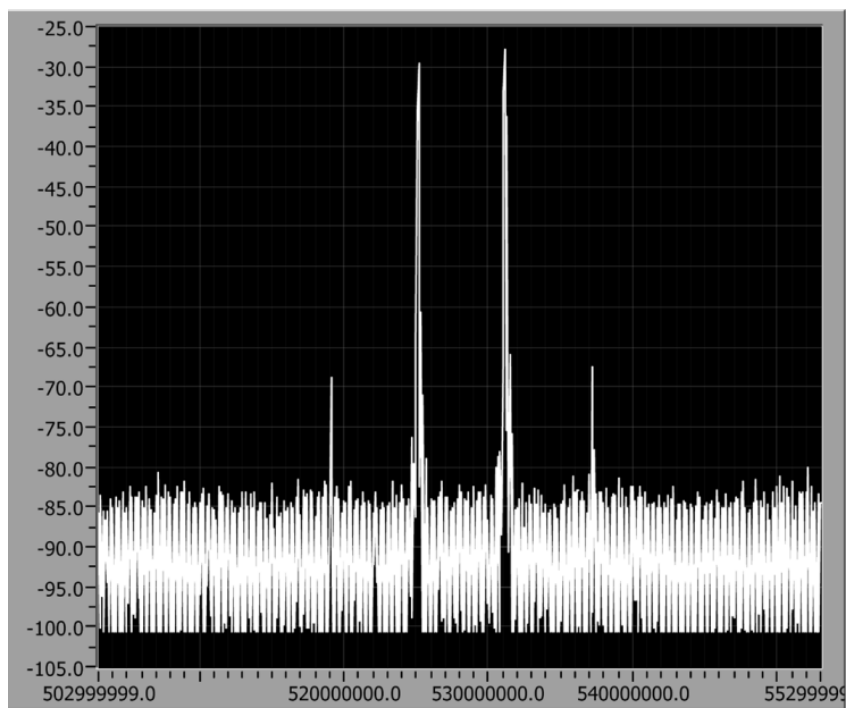

Fig. 14. Output of the spectrum analyzer for RF input frequencies of 5.013 and $5.019 \mathrm{GHz}$ and $\mathrm{RF}$ input power of $-10 \mathrm{dBm}$.

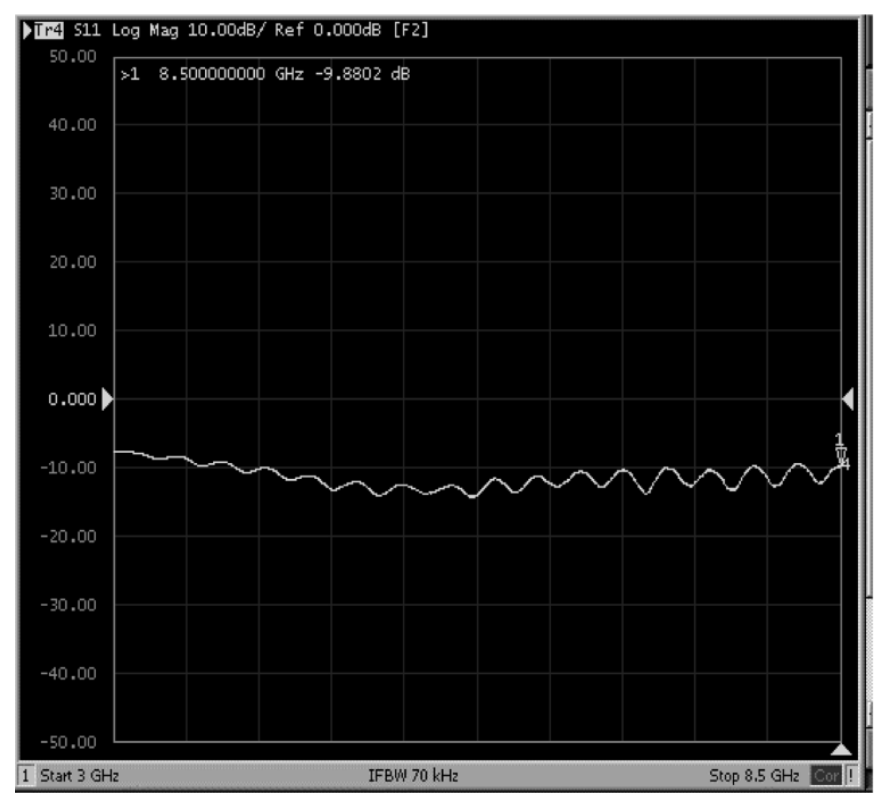

Fig. 15. Magnitude of input reflection coefficient at the RF port.

The noise measurement of the proposed distributed mixer was carried out using the HP8970S. Fig. 16 demonstrates the measured NF versus frequency. The measured NF varied from 6.8 to $7.3 \mathrm{~dB}$ over the entire $7.5-\mathrm{GHz}$ bandwidth.

Table I compares the performance of the proposed mixer circuit with that of previous works.

\section{CONCLUSION}

The design of a novel CMOS distributed mixer with $0.18-\mu \mathrm{m}$ technology was demonstrated. A wide range of the frequency down converting was accomplished using this circuit. This circuit showed a high and flat conversion gain, and a good linearity over up to the $8.72 \mathrm{GHz}$. The proposed circuit also achieved a good matching up to $8.72 \mathrm{GHz}$. This mixer can be employed in RF front-end of multiband UWB receivers.

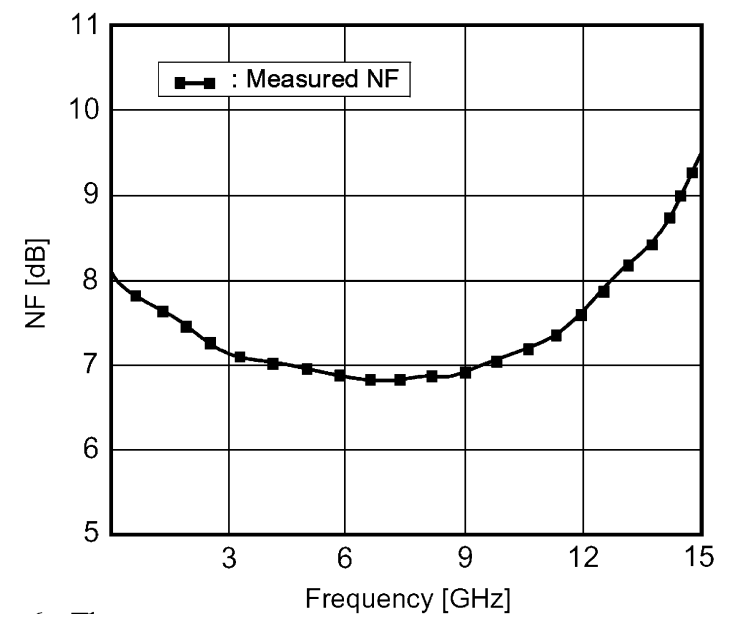

Fig. 16. Measured NF of the proposed two-stage distributed mixer circuit.

TABLE I

Performance Comparison Between Previously Published Works on WidebAND MiXers AND THE PROPOSED Distributed MiXER

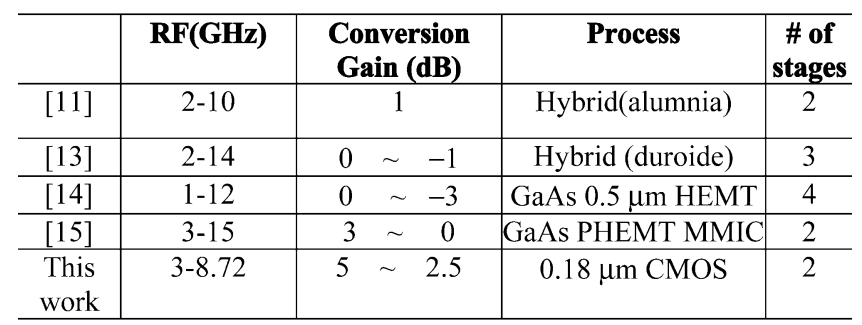

\section{ACKNOWLEDGMENT}

The authors would like to thank Broadcom, Inc., and in particular, M. Isaacs, for providing the measurement equipments. The authors would also like to thank the Sintegrity Inc., for designing and assembling the testing board, and Jazz Semiconductor, Inc., Newport Beach, CA, for providing the device and simulation data, particularly, M. Racanelli and P. Colestock for their help and support.

\section{REFERENCES}

[1] H. Wu and A. Hajimiri, "Silicon-based distributed voltage-controlled oscillators," IEEE J. Solid-State Circuits, vol. 36, no. 3, pp. 493-502, Mar. 2001.

[2] N. T. Terrovitis and R. G. Meyer, "Noise in current-commuting CMOS mixers," IEEE J. Solid-States Circuits, vol. 34, no. 6, pp. 772-783, Jun. 1999.

[3] K. L. Deng and H. Wang, "A 3-33 GHz PHEMT MIMIC distributed mixer," in Proc. IEEE RF Integrated Circuits Symp., 2002, pp. 151-154.

[4] M. Lacon, K. Nakano, and G. S. Dow, "A wide band distributed dual gate HEMT mixer,” in Proc. GaAs IC Symp. Dig., 1988, pp. 173-176.

[5] H. T. Ahn and D. J. Allstot, "A 0.5-8.5 GHz fully differential CMOS distributed amplifier," IEEE J. Solid-State Circuits, vol. 37, no. 8, pp. 985-993, Aug. 2002.

[6] B. M. Ballweber, R. Gupta, and D. J. Allstot, "A fully integrated 0.5-5.5-GHz CMOS distributed amplifier," IEEE J. Solid-State Circuits, vol. 35, no. 2, pp. 231-239, Feb. 2000.

[7] P. Heydari and M. Pedram, "Analysis and reduction of capacitive coupling noise in high-speed VLSI circuits," in IEEE Int. Conf. Comput. Design, Austin, TX, Sep. 2001, pp. 104-109.

[8] B. Kleveland, C. H. Diaz, D. Vook, L. Madden, T. H. Lee, and S. S. Wong, "Exploiting CMOS reverse interconnect scaling in multigigahertz amplifier and oscillator design," IEEE J. Solid-State Circuits, vol. 36 , no. 10 , pp. $1480-1488$, Oct. 2001. 
[9] J. B. Beyer, S. N. Prasad, R. C. Becker, J. E. Nordman, and G. K. Hohenwarter, "MESFET distributed amplifier design guidelines," IEEE Trans. Microw. Theory Tech., vol. MTT-32, no. 3, pp. 268-275, Mar. 1984.

[10] T. H. Lee, The Design of CMOS Radio-Frequency Integrated Circuits. Cambridge, U.K.: Cambridge Univ. Press, 1998.

[11] O. Tang and C. S. Aitchison, "A very wide-band microwave mixer using the distributed mixing principle," IEEE Trans. Microw. Theory Tech., vol. MTT-33, no. 12, pp. 1470-1478, Dec. 1985.

[12] T. Y. Wong, Fundamentals of Distributed Amplification. Norwood, MA: Artech House, 1993.

[13] A. H. Darsinooieh and O. Palamutcuoglu, "On the theory and design of subharmonically drain pumped microwave MESFET distributed mixers," in Proc. 8th Mediterranean Electrotechnical Conf., (MELECON), vol. 1, May 1996, pp. 595-598.

[14] T. S. Howard and M. Paivo, "A distributed 1-12 GHz dual-gate FET mixer," in Proc. IEEE MTT-S Dig., vol. 86, Jun. 1986, pp. 329-332.

[15] I. D. Robertson and A. H. Aghvami, "A novel 1 to $14 \mathrm{GHz}$ monolithic matrix distributed FET mixer," in Proc. 21st Eur. Microwave Conf., Sep. 1991, pp. 489-494.

[16] C. S. Aitchison, "The intrinsict noise figure of the MESFET distributed amplifier," IEEE Trans. Microw. Theory Tech., vol. MTT-33, no. 6, pp. 460-466, Jun. 1985.

[17] G. R. Aiello, "Challenges for ultrawide-band (UWB) CMOS integration," in Proc. IEEE MTT-S, vol. 1, 2003, pp. 361-364.

[18] A. Papoulis, Probability, Random Variables, and Stochastic Processes. New York: McGraw-Hill, 1991.

[19] Y. Tsividis, Operation \& Modeling of the MOS Transistor. New York: McGraw-Hill, 1999.

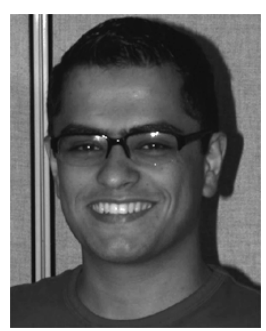

Amin Q. Safarian (S'05) received the B.S. and M.S. degrees in electrical engineering from Sharif University of Technology, Tehran, Iran, in 2000 and 2002 , respectively. He is currently working toward the Ph.D. degree at the University of California, Irvine.

His research interest is to design RF integrated circuit for wireless communication transceivers.

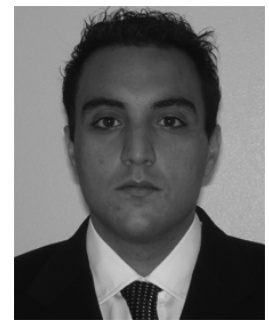

Ahmad Yazdi (S'02) was born in April 1978 in Tehran, Iran. He received the B.S. degree from Sharif University of Technology, Tehran, Iran, in 2001 and the M.S. degree (hons.) from the University of Southern California, Los Angeles, in May 2003, both in electrical engineering with emphasis in communication science. He is currently working toward the Ph.D. degree in broadband circuit design at University of California, Irvine.

Dr. Yazdi is the recipient of the Ph.D. Fellowship Award from Center for Pervasive Communications and Computing in 2003. He is also a member of Etta Kappa Nu.

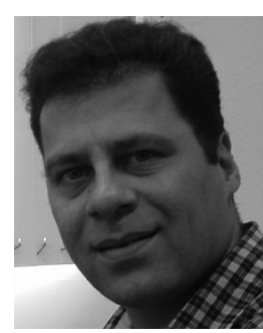

Payam Heydari (S'98-M'00) received the B.S. degree in electronics engineering and the M.S. degree (hons.) in electrical engineering from the Sharif University of Technology, Tehran, Iran, in 1992 and 1995, respectively. He received the Ph.D. degree in electrical engineering at the University of Southern California, Los Angeles, in 2001

During the summer of 1997, he was with Bell-Labs, Lucent Technologies, Holmdel, NJ, where he worked on noise analysis in deep submicron VLSI circuits. He worked at IBM T. J. Watson Research Center, Yorktown Heights, NY, on gradient-based optimization and sensitivity analysis of custom integrated circuits during the summer of 1998 Since August 2001, he has been an Assistant Professor of electrical engineering at the University of California, Irvine, where his research interests are design of high-speed analog, RF, and mixed-signal integrated circuits, and analysis of signal integrity and high-frequency effects of on-chip interconnects in high-speed VLSI circuits. He has published more than 35 conference and journal papers.

Dr. Heydari is the recipient of the 2005 National Science Foundation (NSF) CAREER Award. He has also received the Best Paper Award at the 2000 IEEE International Conference on Computer Design (ICCD). He has also received the Technical Excellence Award from the Association of Professors and Scholars of Iranian Heritage in California in 2001. He serves as a member of the Technical Program Committees of the IEEE Design and Test in Europe (DATE), the International Symposium on Physical Design (ISPD), the International Symposium on Quality Electronic Design (ISQED), and the International Symposium on Low-Power Electronics and Design (ISLPED). 